\title{
Dissolved Organic Matters Impact on Colour Reconstruction in Underwater Images
}

\author{
J. Åhlén ${ }^{1}$, D. Sundgren ${ }^{2}$, T. Lindell ${ }^{3}$, and E. Bengtsson ${ }^{3}$ \\ 1 Centre for Image Analysis, Uppsala University \\ Kungsbäcksv. 47, 80176 Gävle, Sweden \\ jae@hig.se \\ http://www.cb.uu.se \\ 2 Department of Computer and Systems Sciences, \\ Royal Institute of Technology, \\ Kungsbäcksv. 47, 80176 Gävle, Sweden \\ dsn@hig.se \\ 3 Centre for Image Analysis, Uppsala University, \\ Lägerhyddsvägen 3, 75237 Uppsala, Sweden \\ \{tommy, ewert\}@cb.uu.se \\ http://www.cb.uu.se
}

\begin{abstract}
The natural properties of water column usually affect underwater imagery by suppressing high-energy light. In application such as color correction of underwater images estimation of water column parameters is crucial. Diffuse attenuation coefficients are estimated and used for further processing of underwater taken data. The coefficients will give information on how fast light of different wavelengths decreases with increasing depth. Based on the exact depth measurements and data from a spectrometer the calculation of downwelling irradiance will be done. Chlorophyll concentration and a yellow substance factor contribute to a great variety of values of attenuation coefficients at different depth. By taking advantage of variations in depth, a method is presented to estimate the influence of dissolved organic matters and chlorophyll on color correction. Attenuation coefficients that depends on concentration of dissolved organic matters in water gives an indication on how well any spectral band is suited for color correction algorithm.
\end{abstract}

\section{Introduction}

Colour correction of underwater images mainly focuses on balancing colours where blue colour can be prevailing [1]. The bluishness in images caused by apparent optical properties of water where light in the red and yellow region of the spectra will be more attenuated with increasing depth than in the blue spectral region [2]. Underwater photographers which are concerned with more natural color representation in the images use filters, but filters cannot fix color problems when shooting in ambient light at depths where red and yellow wavelengths of light are almost totaly attenuated [3]. Underwater photographers usually set 
up a pair of strobes on the camera as an extra light source. The drawbacks are though that the subject may be too large for conventionally mounted strobes, or the photographer working with strobes at depth may be unable to approach the subject close enough to accommodate the limited illumination range of strobe lights [4]. In either case, the result is that the subject is illuminated solely by ambient light in which red and yellow wavelengths may be deficient or totally absent.

The camera reproduces the wavelengths of light that enter the lens as faithfully as the design of the film emulsion or CCD sensor allows. Some cameras includes beautifying functions that adapt to the dominating light spectrum and filter the intensity values that have been registrated. Based on intensity values of the image we can estimate the downwelling irradiance and diffuse attenuation coefficients $K_{d}$ of the diving site. Strictly speaking, $K_{d}$ is not a property of the water itself but rather a descriptor of the underwater light field that varies with depth, solar altitude, and time. The reconstruction of colour in the image is based on the spectral model of the acquisition system and a gray reflectance target, which is present in each image [5]. By inverting the model, an estimation of the spectral reflectance of each pixel is possible. The reflectance of an object can be considered as a ratio of the intensity of reflected radiant energy to that reflected from a defined gray reference standard. In image collection and further processing the authors are using a gray Spectralon with $99 \%$ reflectance as such a standard [6].

Estimated values of $K_{d}$ values gives a possibility to calculate the absorption coefficients of dissolved organic matters and chlorophyll for that particular water. We are arguing that the absorption coefficients can give an indication on what $K_{d}$ values should be used in color reconstruction of the images.

Not only the pure water itself will affect the optical climate of the water mass and affect the colour in the photos taken at different water depths, but also all other inherent optical properties will have impact, sometimes substantial. So far our work has mainly been dealing with correction for the attenuation of the water itself. In a certain environment it could be recommendable to colour correct or at least be aware of possible effects of substances in the water.

\subsection{Dissolved Matters and Chlorophyll}

Two essentially different types of substances or substance behaviour need to be discussed. We separate between dissolved and suspended matter. The dissolved matter is commonly of organic origin and usually named yellow substance. Also the salt in sea water could have some influence of the optical behaviour. The suspended matter could be either inorganic or organic and the organic matter could be dead or living. It is common to call the inorganic matter just suspended matter and it is also common to treat the phytoplankton separately, due to its water quality importance.

The inorganic matter has a rather well defined behaviour, mainly as a matter of particle size distribution and the influence of dissolved matter is also rather easy to model. Phytoplankton influence, however, is very complex and the com- 
plexity is much worse due to the fact that we always have a mixture of all three agents in natural waters.

In situ spectral absorption coefficient profiles can be measured with spectral radiometers [7]. This means that in order to calculate the spectral absorption of a particular water layer it is enough to measure a spectra with spectrometer. The instrument we use includes 1024 spectral channels and register pointwise the intensity counts. The concentration of dissolved organic matter is usually based on the amount of organic carbon present in the water. Experiments have shown that the spectral absorption of yellow substance varies approximately exponentially with wavelength and increases with decreasing wavelength [2]. Phytoplankton specific absorption coefficients have two absorption peaks at 440 and $675 \mathrm{~nm}$. The peak at $440 \mathrm{~nm}$ is stronger than at $675 \mathrm{~nm}$ [8]. In clear near shore ocean waters the concentration of non-organic suspended matters is predominant, however we are not concerned with the calculation of those. Concentration of dissolved organic matters and chlorophyll may vary greatly for different diving sites thus should be measured in situ or estimated from measured irradiances.

Underwater photos are most commonly taken in a coral reef environment and here we often have very clear water with little material, suspended as well as dissolved. Typically there are very low concentrations of small fractions of inorganic matter. Only occasionally a plankton bloom may occur. Our colour correction model [5] is therefore often sufficient for reefs photos.

As we have taken photos in Florida, Portugal and Sweden we do need to, at least theoretically, discuss the colour effects of substances in the water as suspended matter, phytoplankton and yellow substances are very common in those waters, typically a mixture of all three. In this paper we will discuss the combined effects of dissolved matter and phytoplankton. The influence of suspended matter will be discussed in a separate paper.

\section{Estimating the Absorption Coefficient for Dissolved Matters and Chlorophyll}

One way to express the concentration of dissolved organic matters in water is to model the absorption property of the matter. In phytoplankton-dominated waters near the sea surface, the total absorption and backscattering coefficients can be written in terms of chlorophyll concentration $C$ and yellow substance factor $Y$ for each depth interval, see Eq. 1.

$$
a(\lambda, z)=a_{w}(\lambda)+0.06 a_{p h}^{*}(\lambda) C(z)^{0.65}+Y(z) a_{p h}(440) \exp (-0.014(\lambda-440))
$$

From this equation it can be seen that $Y$ describes the relationship between the absorption by yellow substance at $440 \mathrm{~nm}$ and the absorption by phytoplankton at $440 \mathrm{~nm}$.

The equations 2 and 3 for concentration of chlorophyll and yellow substances below are from [8]. Eq. 2

$$
C=\left(\left(\delta_{j i} K_{d}-0.686 \delta_{s t} K_{d}+0.0753\right) / 0.0746\right)^{1.54}
$$


and $Y$ Eq. 3

$$
Y=-\left(\delta_{j i} K_{d}+0.0049-0.0107 C^{0.65}\right) / 0.0625 C^{0.65},
$$

where $\delta_{j i} K_{d}=K\left(\lambda_{j}, z z_{1}\right)-K\left(\lambda_{i}, z z_{1}\right)$. An analysis of the commonly used wavelengths in ocean color measurements showed that the optimal wavelength pairs to solve these equations are $(412,443)$ and $(443,555)[8]$. The $a_{w}(\lambda)$ and $a_{p h}^{*}$ coefficients are commonly used and found in $[9,10] . K_{d}$ values are estimated from intensity count measurements with the spectrometer at different depths.

\section{Correction of Underwater Images}

The general method requires the presence of the gray standard in each photographed scene. However for more accurate estimation of the attenuation coefficients we are forced to further measurements with the spectrometer. Apart from that we apply the correct spectra on the images in order to remove all the beautifying effects built into the camera so that the image is as correct as possible before it is subjected to colour correction algorithm. Test imagery was collected off the coast of Lisbon at Sisimbra in April 2004. For each image we recorded the exact depth at which it was taken. To diminish the water absorption effects we use Beer's Law [11] to calculate the new intensity values for the image taken at depth $z$ as if it was taken at depth $z_{1}$. The method consists of these steps:

1. We are using a spectrometer for this study and measure the intensity counts when pointing to the gray reflectance plate. We can then use the known reflectance of the gray plate to extrapolate the downwelling irradiance to the depth in question. Downwelling irradiance is proportional to intensity counts that we have from spectrometer, which means that the ratio between two downwelling irradiances for different wavelengths is equal to the ratio between two corresponding intensity counts.

2. We calculate $K_{d}$ values from estimated downwelling irradiances for each depth interval by using the following equation:

$$
K_{d}\left(\lambda_{i}, z z_{1}\right)=\frac{I\left(\lambda_{i}\right)-I\left(\lambda_{j}\right)}{z z_{1} I\left(\lambda_{i}\right)},
$$

as can be seen in Figure 1.

3. The variation of downwelling irradiance with depth can be approximated by

$$
I\left(z_{1}\right)=I(z) e^{K_{d}(z)-K_{d}\left(z_{1}\right) z_{1}-z} .
$$

4. Each pixel for red, green and blue components of the image is subject to this operation and as a result we "lift up" the image to depth $z_{1}$. For this operation we choose three $K_{d}$ values for the three spectral channels red, green and blue. 


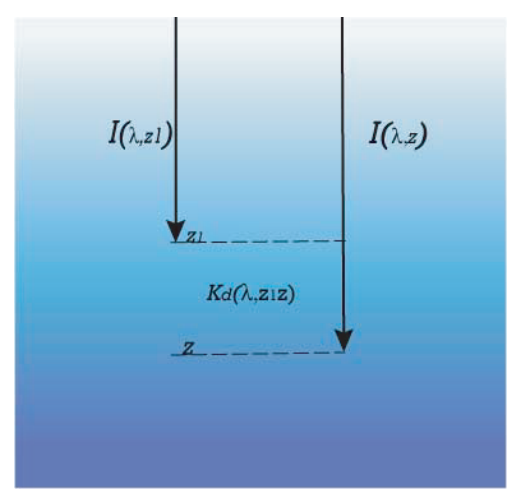

Fig. 1. Illustration of Beer's Law

\section{Results}

By modeling the absorption behaviour for each available wavelength we can clearly see which wavelengths are more sensitive to the absorption of dissolved organic matters in the water. In Figures 2, 3 and 4 we see the $K_{d}$ values and absorption coefficients, respectively, plotted as functions of wavelength. As expected the shape of curves for the absorption coefficients shows the presence of phytoplankton in the water. The points marked with $\times$ show which wavelength represents red, green and blue, respectively. We can see clearly that $K_{d}$ values representing
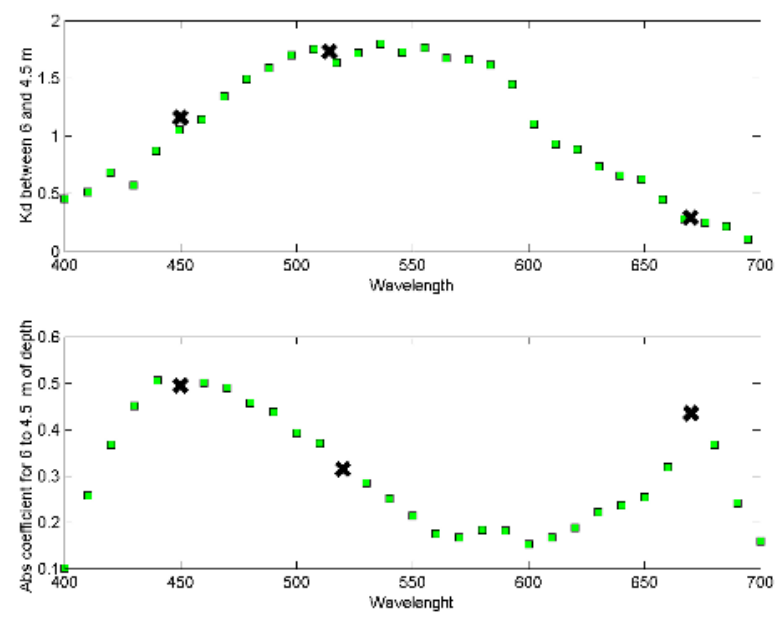

Fig. 2. $K_{d}$ used for correction of colors in images at depths between $6 \mathrm{~m}$ and $4.5 \mathrm{~m}$ compared to absorption coefficients 

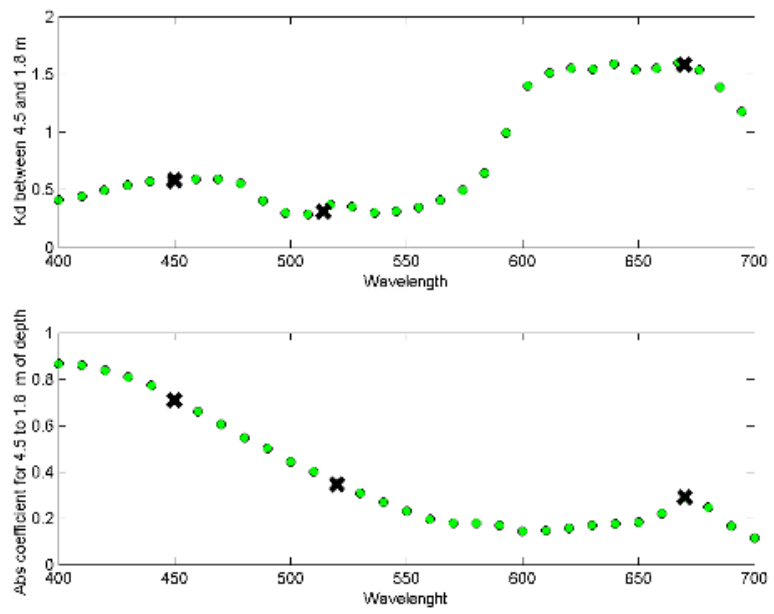

Fig. 3. $K_{d}$ used for correction of colors in images at depths between $4.5 \mathrm{~m}$ and $1.8 \mathrm{~m}$ compared to absorption coefficients
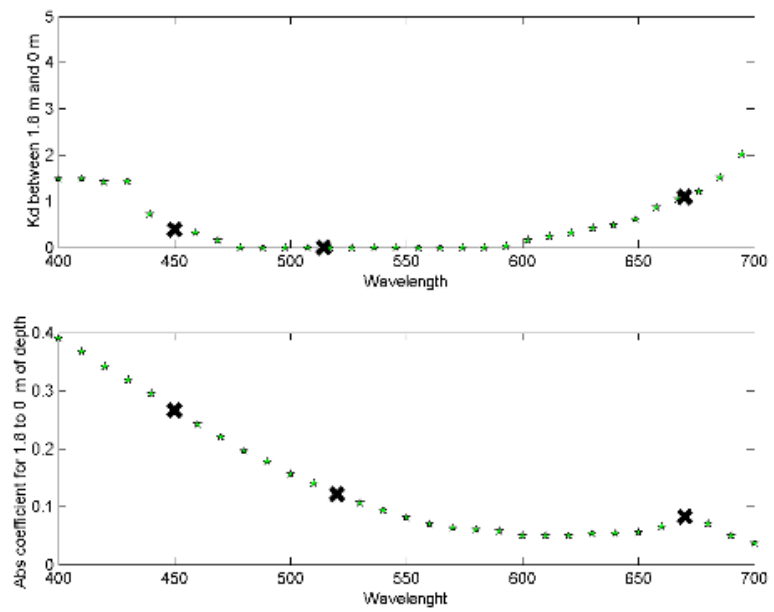

Fig. 4. $K_{d}$ used for correction of colors in images at depths between $1.8 \mathrm{~m}$ and $0 \mathrm{~m}$ compared to absorption coefficients

the red spectral interval correspond to the same wavelength as the lower peak value of absorption coefficient. This is illustrated especially clear in Figure 2.

In the graphs we can see that the value for the red channel corresponds to one of the absorption peaks. We set the marked values in the color reconstruction algorithm. The image that is taken at depth of 4.5 meters was corrected as to show what it would have looked like had it been taken at 1.8 meters depth, see Figures 5 and 6. 


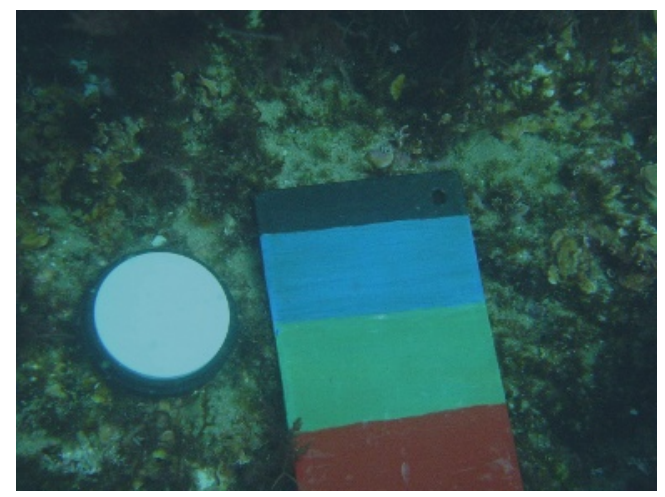

Fig. 5. Original image taken at 4.5 meters of depth

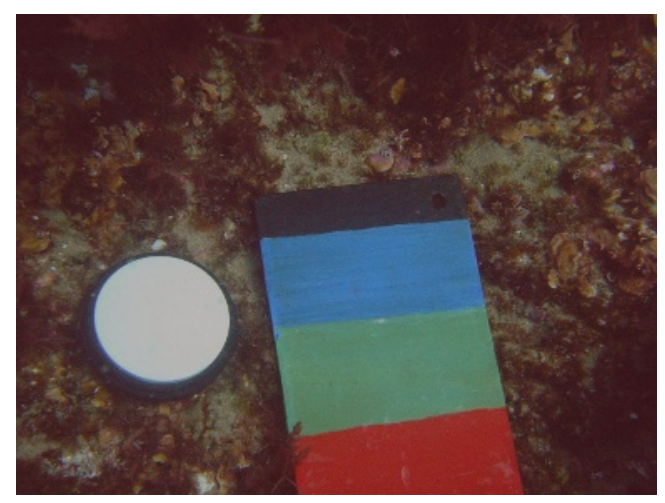

Fig. 6. Corrected image

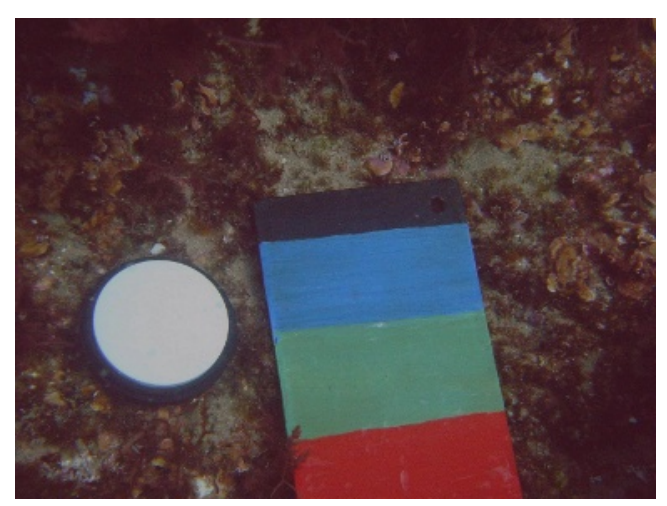

Fig. 7. Corrected image with new $K_{d}$ value for the red channel 
To avoid the influence of phytoplankton when red channel is color corrected we choose another value of $K_{d}$ that does not correspond to the peak of total absorption coefficient and also correspond to the least rate of change in the red channel. Experiments have shown that the best value to be set into Beer's law for red channel is 1.56 instead of 1.60. The result is shown in Figure 7.

\section{Conclusions}

The $K_{d}$ values that are used in the color correction algorithm can be chosen based on the concentration of dissolved organic matters in the particular water layer. The red channel (the interval between 600 and $700 \mathrm{~nm}$ ) is the most sensitive for underwater imaging therefore we should take into account every possible source of noise in this channel. In order to choose the least sensitive $K_{d}$ value for colour correction we consider both of the following points:

- The representative wavelength for the red channel should not be in the neighborhood of the one that corresponds to the peak value of the total absorption coefficient.

- The rate of change for the $K_{d}$ curve should be minimal in the red interval.

Since environmental differences will result in variations of optical water properties we need to find $K_{d}$ values for each diving site. From those estimated values we have to choose three that represent the red, green and blue spectral intervals. We have found an indicator on what $K_{d}$ values should be chosen for color correction. This will reduce the time needed to investigate and test the different $K_{d}$ values.

For this study we would benefit from images and spectrometer data taken at depths with smaller interval. This would most likely give us less varying $K_{d}$.

We tested the method on the images taken in Mid Atlantic where corals are absent. In such waters the dissolved organic matters and chlorophyll are predominant, but scarce. Therefore the change of $K_{d}$ values did not have a dramatic effect on colour correction. In the next stage of this project we would model the influence of color reconstruction by presence of suspended non-organic matters which usually dominate water column above coral reefs.

\section{Acknowledgments}

We would like to thank KK-foundation for the financial support for this project.

\section{References}

1. Fissenko, T., Y., Fissenko, V., T., Rogatchev, G., A. and Sushechev. G., A. An interpolation method for color image reconstruction in deep underwater observation. In: Proc. of D. S. Rozhdestvensky Optical Society: The II International Conference, Current Problems in Optics of Natural Waters, St.Petersburg, Russia, 2003, pp. 113-118. 
2. R.E. Walker, Marine Light Field Statistics, John Wiley \& Sons, Inc, 1994.

3. R. Delfs, Getting Rid of the Underwater Blues, Wetpixel.com, url: http://www.wetpixel.com, February 2005.

4. A. Kohler, D. Kohler, Underwater Photography Handbook. UK London: New Holland Publishers, 1998.

5. J. Åhlén and E. Bengtsson and T. Lindell, Color Correction of Underwater Images Based on Estimation of Diffuse Attenuation Coefficients, In Proceedings of the PICS Conference An International Technical Conference on The Science and Systems of Digital Photography including the Fifth International Symposium on Multispectral Color Science, Rochester, NY, 13-16 May 2003.

6. Spectralon, Reflectance Material for Component Fabrication, Labsphere url: http://www.labshere.com/products/Products.asp, January, 2005.

7. N.K. Hojerslev, A spectral light absorption meter for measurements in the sea. 1975 In: Limnol. Oceanogr., 20: 1024-1034.

8. J. S. Bartlett, M. R. Abbott, R. M. Letelier and J. G. Richman, Chlorophyll Concentration Estimated From Irradiance Measurements At Fluctuating Depths, In: Ocean Optics XIV, Kailua-Kona, November 1998.

9. L. Prieur and Sathyendranath, An optical classification of coastal and oceanic waters based on the specific spectral absorption curves of phytoplankton pigments dissolved organic matter and other particulate materials for 400-700 nm, In Limnol. Oceanogr, vol. 26(4), 1981, pp. 671-689.

10. R. C. Smith and K. S. Baker, Optical properties of the clearest natural waters (200-800) nm, In Appl. Opt, vol. 20, 1981 pp.177-184.

11. Calculations Using Beer's Law, url: http://www.oceansonline.com/beers_law.htm, (November 2002) 